

\section{Water-Quality Data for Navajo National Monument, Northeastern Arizona-2001-02}

Open-File Report 03-287

Prepared in cooperation with the

NATIONAL PARK SERVICE 


\section{U.S. DEPARTMENT OF THE INTERIOR \\ GALE A. NORTON, Secretary}

U.S. GEOLOGICAL SURVEY

Charles G. Groat, Director

The use of firm, trade, and brand names in this report is for identification purposes only and does not constitute endorsement by the U.S. Geological Survey.

For additional information write to:

District Chief

U.S. Geological Survey

Water Resources Division

520 N. Park Avenue, Suite 221

Tucson, AZ 85719-5035
Copies of this report can be purchased from:

U.S. Geological Survey

Information Services

Box 25286

Federal Center

Denver, C0 80225-0046

Information about U.S. Geological Survey programs in Arizona is available online at http://az.water.usgs.gov. 


\section{CONTENTS}

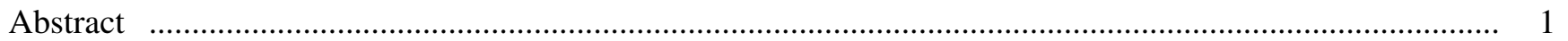

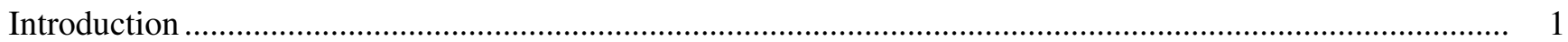

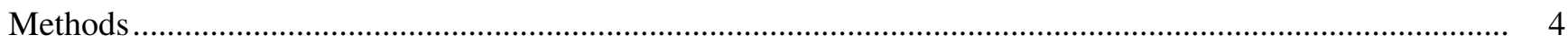

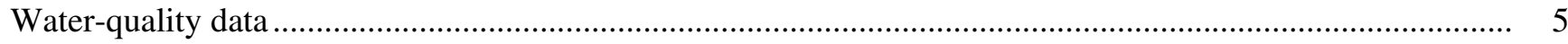

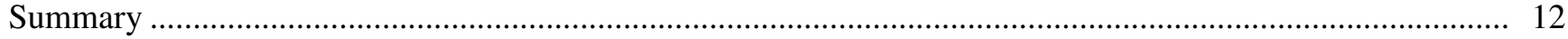

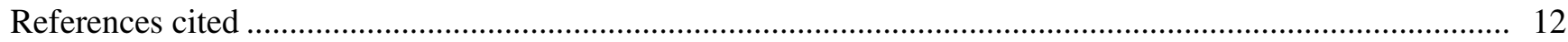

\section{FIGURES}

1. Locations of Navajo Monument and water-quality data-collection sites.......................................... 2

\section{TABLES}

1. Location, altitude, and hydrologic characteristics of water-quality data-collection sites,

Navajo National Monument, Arizona, 2001-02

2. Field measurements, and concentrations of major ions, nutrients, trace elements, and uranium in water samples from selected sites, Navajo National Monument, Arizona, 2001-02

3 U.S. Environmental Protection Agency Primary and Secondary Maximum Contaminant Levels for analyzed constituents in water samples from selected sites, Navajo National Monument, Arizona, 2001-02

4. Discharge at selected sites, Navajo National Monument, Arizona, 2001-02

\section{CONVERSION FACTORS AND DATUMS}

\begin{tabular}{lcl}
\hline Multiply & \multicolumn{1}{c}{ By } & To obtain \\
\hline inch (in) & 2.54 & centimeter \\
inch (in) & 25.4 & millimeter \\
foot (ft) & 0.3048 & meter \\
mile (mi) & 1.609 & kilometer \\
square mile $\left(\mathrm{mi}^{2}\right)$ & 2.590 & square kilometer \\
acre-foot $(\mathrm{acre}-\mathrm{ft})$ & 0.001233 & cubic hectometer \\
cubic foot per second $(\mathrm{ft} / \mathrm{s})$ & 0.02832 & cubic meter per second \\
gallon per minute $(\mathrm{gal} / \mathrm{min})$ & 0.06309 & liter per second \\
gallon per day (gal/d) & 0.003785 & cubic meter per day \\
\hline
\end{tabular}

Temperature in degrees Celsius $\left({ }^{\circ} \mathrm{C}\right)$ may be converted to degrees Fahrenheit $\left({ }^{\circ} \mathrm{F}\right)$ as follows:

$$
{ }^{\circ} \mathrm{F}=\left(1.8^{\circ} \mathrm{C}\right)+32
$$


Vertical coordinate information is referenced to the National Geodetic Vertical Datum of 1929 (NGVD 29)—a geodetic datum derived from a general adjustment of the first-order level nets of both the United States and Canada, formerly called Sea Level Datum of 1929; horizontal coordinate information is referenced to the North American Datum of 1927 (NAD 27). Altitude, as used in this report, refers to distance above or below NGVD 29.

\section{ABBREVIATED WATER-QUALITY UNITS}

Chemical concentration and water temperature are given only in metric units. Chemical concentration in water is given in milligrams per liter $(\mathrm{mg} / \mathrm{L})$ or micrograms per liter $(\mu \mathrm{g} / \mathrm{L})$. Milligrams per liter is a unit expressing the solute mass (milligrams) per unit volume (liter) of water. One thousand micrograms per liter is equivalent to 1 milligram per liter. For concentrations lower than 7,000 milligrams per liter, the numerical value is about the same as for concentrations in parts per million. Specific conductance is given in microsiemens per centimeter at 25 degrees Celsius $\left(\mu \mathrm{S} / \mathrm{cm}\right.$ at $\left.25^{\circ} \mathrm{C}\right)$. 


\title{
Water-Quality Data for Navajo National Monument, Northeastern Arizona—2001-02
}

\author{
By Blakemore E. Thomas
}

\begin{abstract}
Water-quality data are provided for six sites in Navajo National Monument in northeastern Arizona. These data describe the current water quality and provide baseline water-quality information for monitoring future trends.

Water samples were collected from six sites near three ancient Indian ruins during September 2001 to August 2002. Two springs and one well are near Betatakin Ruin, one spring is near Keet Seel Ruin, and one spring and one stream are near Inscription House Ruin. Water from all the sites is from the $\mathrm{N}$ aquifer, a regional sandstone aquifer that is the source of drinking water for most members of the Navajo Nation and Hopi Tribe in northeastern Arizona.

Concentrations of dissolved solids, major ions, trace elements, and uranium were low at the six sites. Dissolved-solids concentration ranged from 94 to 221 milligrams per liter. Concentrations of dissolved nitrate (as nitrogen) were generally low (less than 0.05 to 0.92 milligrams per liter) and were within the range of concentrations at other $\mathrm{N}$-aquifer sites within 20 miles of the study area. Water samples from Inscription House Spring, Navajo Creek Tributary (near Inscription House Ruin), and Keet Seel Ruin Spring contained indicators of human or animal wastes-fecal coliform and Escherichia coli bacteria.
\end{abstract}

\section{INTRODUCTION}

In 1992, the National Park Service established a nationwide Level 1 Water Quality Inventory and Monitoring Program to obtain baseline water-quality information and to assess possible water-quality problems in national parks and monuments throughout the United States. Key water bodies are identified that are essential to the cultural, historical, or natural resource management themes or that provide habitats for threatened or endangered plants and animals. The basic chemical character and possible waterquality problems of the water bodies are described through the collection and analysis of water samples and assessment of the chemical data. In 2001, the U.S. Geological Survey (USGS), in cooperation with the National Park Service, began studies of Navajo, Walnut Canyon, and Wupatki National Monuments in northeastern Arizona as part of this Level I program. This report describes results of a water-quality inventory of Navajo National Monument (fig. 1).

Navajo National Monument was established in 1909 to protect and preserve three ancient Indian ruins that were occupied from about 1250 to 1300 . In addition to the ruins, the monument is required to protect the area's natural resources. The water resources of the monument are used for public drinking-water supply and to support the natural plant and animal populations. Monument employees have a need for a better understanding of the quality of water resources for effective and informed management of visitor use and natural-resource protection. With the exception of a public-supply well, there are no historical water-quality data sites within the monument, and little is known about water quality in the area. 


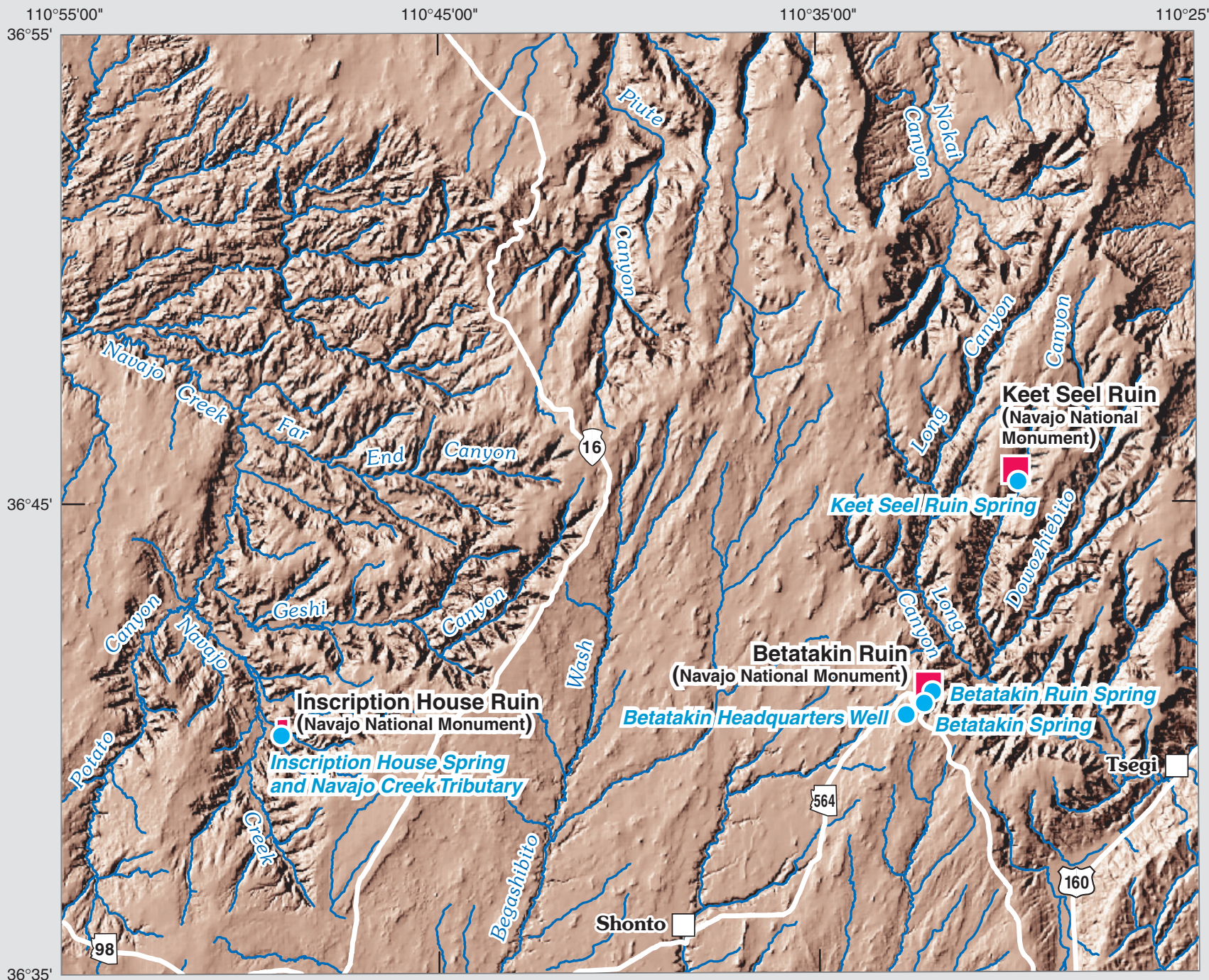

Base from U.S. Geological Survey

digital elevation model data,

Universal Transverse Mercator

Projection, Zone 12. Datum: NAD 27

\begin{tabular}{|cccc}
0 & 5 & & 10 \\
0 & 1 & 1 & 1
\end{tabular}

EXPLANATION

WATER-QUALITY DATA-COLLECTION SITE

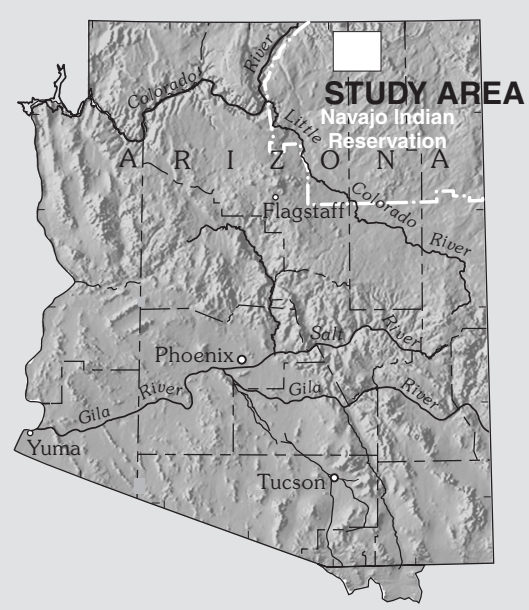

Figure 1. Locations of Navajo Monument and water-quality data-collection sites. 
The objectives of this water-quality inventory of Navajo National Monument were to identify the key water bodies in the monument, to describe the current water-quality conditions, and to assess possible waterquality problems. Water samples were collected from six sites during September 2001 to August 2002. These samples were analyzed for general chemical characteristics and constituents that can be indicators of water-quality problems.

The headquarters of Navajo National Monument is near Betatakin Ruin. Keet Seel Ruin is about 5 miles northeast of Betatakin Ruin, and Inscription House Ruin is about 16 miles west of Betatakin. Three separate monument areas enclose the ruins (fig. 1). The monument area at Betatakin Ruin is 0.25 square mile and ranges in altitude from 6,500 to 7,300 ft. The monument area at Keet Seel Ruin is 0.25 square mile and ranges in altitude from 6,700 to $7,500 \mathrm{ft}$ (fig. 1). The monument area at Inscription House Ruin is 0.0625 square mile and ranges in altitude from 5,400 to $5,900 \mathrm{ft}$ (fig. 1). The three ruins are in canyons that are deeply incised into horizontal layers of sandstone and siltstone.

The general area of the ruins has an arid climate. Precipitation falls mostly as snow in the winter and as rain during the other seasons. Average annual precipitation ranges from about 10 inches at Inscription House Ruin to 12 inches at the monument headquarters at Betatakin (U.S. Department of Agriculture, 1999). Precipitation is spread fairly evenly throughout the four seasons; about 20 percent falls in the spring and about 30 percent falls in the summer (Western Regional Climate Center, Desert Research Institute, 2002).

Frontal storms move through the area in the fall, winter, and spring. Thunderstorms are common in the summer. Most of the vegetation is piñon and juniper trees; greasewood and sagebrush are found in altitudes below $5,500 \mathrm{ft}$.

Important water bodies in the monument include ground water, springs, and perennial and intermittent streams. Ground water is used for drinking water by monument employees and visitors. Springs and streams provide important habitat for plants and animals and drinking water for stock and wild animals. In addition to the current importance to the water resources of the monument, the springs and streams also were the source of drinking and irrigation water for the ancient inhabitants of the area in the 1200s.

Six key water bodies or sample sites were identified in Navajo National Monument (table 1; fig. 1):
1. Spring near Inscription House Ruin (Inscription House Spring),

2. Stream near Inscription House Ruin (Navajo Creek tributary near Shonto),

3. Betatakin headquarters well,

4. Spring near Betatakin Ruin (Betatakin Spring),

5. Spring at Betatakin Ruin (Betatakin Ruin Spring), and

6. Spring near Keet Seel Ruin (Keet Seel Ruin Spring).

Inscription House Spring and Navajo Creek tributary near Shonto are at the southern boundary of the monument at an altitude of about 5,440 ft. The spring flows out of the Navajo Sandstone and into the stream (Davis and others, 1963). The stream has a drainage area of about $1.5 \mathrm{mi}^{2}$, flows east to west, and enters Navajo Creek, which then flows northward into Lake Powell. Several springs that are east and upstream of Inscription House Ruin contribute water to the stream.

The Betatakin headquarters well is at an altitude of about 7,230 ft and is about 1,500 ft southwest of the headquarters. Betatakin Spring is at an altitude of about $6,800 \mathrm{ft}$ and is about 2,000 ft southwest of Betatakin Ruin. Betatakin Ruin Spring is at an altitude of about $6,600 \mathrm{ft}$ and is about $500 \mathrm{ft}$ southeast of the ruin. Keet Seel Ruin Spring is at an altitude of about $6,700 \mathrm{ft}$ and is at the northern end of the ruin's base.

The four springs and well discharge water from the $\mathrm{N}$ aquifer (Davis and others, 1963). Navajo Creek tributary receives ground-water discharge from the $\mathrm{N}$ aquifer and runoff from rainfall and snowmelt. The $\mathrm{N}$ aquifer is a regional sandstone aquifer that extends for more than $6,000 \mathrm{mi}^{2}$ in northeastern Arizona, and is the source of drinking water for most members of the Navajo Nation and Hopi Tribe in northeastern Arizona (Thomas, 2002a).

The four springs and stream are important perennial sources of water for plants and animals. The water is used for habitat and drinking. In addition, Inscription House Spring and Navajo Creek tributary provide water for local stock animals owned by members of the Navajo Nation. The well at Betatakin headquarters supplies water for drinking and other facilities at the monument.

The primary threat to the water quality in the monument is from sheep and cattle that graze the lands surrounding the monument (I.G. Francisco, Navajo National Monument, oral commun., 2001). Manure from these animals could cause nutrient or 
bacteriological contamination of streams or the $\mathrm{N}$ aquifer. Snowmelt or rainfall runoff could carry manure into streams, and infiltrating and percolating recharge water could carry dissolved material from the manure to the $\mathrm{N}$ aquifer. Another threat to water quality is contamination from human tourists that use the monument for day hikes or overnight camping (I.G. Francisco, Navajo National Monument, oral commun., 2001). Bacteriological or nutrient contamination is possible if the humans are not careful about their waste habits.

The author acknowledges the cooperation and assistance of the employees of Navajo National Monument. The guidance and assistance of monument employees were needed to gain access to remote waterquality sites that were difficult to find.

\section{METHODS}

The current chemical character and water quality of the key water bodies of Navajo National Monument were determined by collecting water samples and analyzing those samples for the following properties or constituents: (1) field measurements, (2) nutrients, (3) major ions, (4) trace elements, and (5) uranium. Field measurements include $\mathrm{pH}$, specific conductance, temperature, dissolved oxygen, alkalinity, fecalindicator bacteria [total coliform, fecal coliform, and Escherichia coli (E. coli)], and discharge rates for springs and streams.

Concentrations of bacteria and nitrogen were used to indicate whether or not animal manure or human wastes have affected the water quality of the monument. In addition to animal or human caused water-quality problems, some natural constituents can be harmful to human health when present at high concentrations (U.S. Environmental Protection Agency, 2002). These potentially harmful constituents include some of the major ions, most of the trace elements, and uranium. Concentrations of all the constituents were compared to current U.S. Environmental Protection Agency Primary and Secondary Maximum Contaminant Levels (U.S. Environmental Protection Agency, 2002).

Water samples were collected from the four springs and one stream in September 2001, March 2002, and August 2002, and were analyzed to assess water quality, including seasonal variations. Only two samples were collected from Betatakin Spring because there was a swarm of bees at the spring in September 2001, and it was unsafe to collect a sample. Only one water sample was collected from the monumentheadquarters well (in September 2001) because water quality in a deep aquifer typically has little to no seasonal variation; recharge water that carries new dissolved constituents has a long travel time, and there are many opportunities for attenuation of the recharge water.

Water samples were collected according to standard USGS protocols described in several USGS Techniques of Water-Resources Investigations Reports (Friedman and Erdmann, 1982; Britton and Greeson, 1988; Koterba and others, 1995; Wilde and others, 1998a,b,c; Wilde and Radtke, 1998; Myers and Wilde, 1999; and Wilde and others, 1999). All laboratory analyses of the water samples were conducted at the USGS National Water-Quality Laboratory (NWQL) according to techniques described in Fishman and Friedman (1989), Faires (1993), Fishman (1993), McLain (1993), Struzeski and others (1996), Garbarino (1999), and Jones and Garbarino (1999).

The water sample from the Betatakin headquarters well was collected from a faucet near the wellhead and ahead of any water treatment such as disinfection, softening, or filtration. Polyethylene tubing was used to connect the faucet to a splitter that directed the water to a flow chamber housing a multiparameter water-quality monitoring probe, to a filter line, to a whole-water line, and to a disposal or overflow line. Temperature, $\mathrm{pH}$, specific conductance, and dissolved-oxygen concentrations were measured in the flow chamber with the monitoring probe, which was calibrated each day of the sampling trip. Water samples were collected from a filtration unit for analysis of dissolved constituents, and water samples were collected directly from the faucet for bacteriological analyses.

The water samples from Navajo Creek tributary were collected as point (grab) samples. Field measurements (temperature, $\mathrm{pH}$, specific conductance, and dissolved oxygen) were made on-site with instruments that were calibrated each day of the sampling trip. Discharge was measured with a 3-inch Parshall flume. 
Table 1. Location, altitude, and hydrologic characteristics of water-quality data-collection sites, Navajo National Monument, Arizona, 2001-02

[N/A, not applicable]

\begin{tabular}{|c|c|c|c|c|c|c|}
\hline Site name & Latitude & Longitude & $\begin{array}{c}\text { Land- } \\
\text { surface } \\
\text { altitude, in } \\
\text { feet above } \\
\text { sea level }\end{array}$ & $\begin{array}{l}\text { Well depth, in } \\
\text { feet below } \\
\text { land surface }\end{array}$ & $\begin{array}{l}\text { Rock formation } \\
\text { or aquifer }^{1}\end{array}$ & Use of water \\
\hline Inscription House Spring & 364013 & 1104911 & 5,440 & N/A & $\begin{array}{l}\text { Navajo } \\
\text { Sandstone }\end{array}$ & $\begin{array}{l}\text { Plants and animals, } \\
\text { stock animals }\end{array}$ \\
\hline $\begin{array}{l}\text { Navajo Creek tributary near Shonto } \\
\text { (near Inscription House Ruin) }\end{array}$ & 364012 & 1104911 & 5,440 & N/A & N/A & $\begin{array}{l}\text { Plants and animals, } \\
\text { stock animals }\end{array}$ \\
\hline Betatakin headquarters well & 364032 & 1103241 & 7,230 & 753 & $\begin{array}{l}\text { Navajo } \\
\text { Sandstone }\end{array}$ & Public supply \\
\hline Betatakin Spring & 364049 & 1103218 & 6,800 & N/A & $\begin{array}{l}\text { Navajo } \\
\quad \text { Sandstone }\end{array}$ & Plants and animals \\
\hline Betatakin Ruin Spring & 364102 & 1103202 & 6,600 & N/A & $\begin{array}{l}\text { Navajo } \\
\quad \text { Sandstone }\end{array}$ & Plants and animals \\
\hline Keet Seel Ruin Spring & 364534 & 1102938 & 6,700 & N/A & $\begin{array}{l}\text { Navajo } \\
\quad \text { Sandstone }\end{array}$ & Plants and animals \\
\hline
\end{tabular}

${ }^{1}$ Davis and others, 1963.

Water samples from the springs were collected as point (grab) samples. Field measurements (temperature, $\mathrm{pH}$, specific conductance, and dissolved oxygen) were made on site with instruments that were calibrated each day of the sampling trip. Discharge from the springs was measured with a 3-inch Parshall flume or volumetrically.

All samples sent to the NWQL were processed and preserved as described in the appropriate protocols. Samples from all sites were passed through a 0.45-micron filter for analyses of dissolved constituents (nutrients, major ions, trace elements, uranium, and alkalinity). Alkalinity was measured in the field using electrometric procedures. Water samples for bacteriological analysis were collected and stored in sterile bottles prior to preparation for standard platecount analysis.

\section{WATER-QUALITY DATA}

The six sites near Betatakin, Keet Seel, and Inscription House Ruins have a calcium bicarbonate water type that is associated with recharge areas for the $\mathrm{N}$ aquifer (table 2; Thomas, 2002a). Water samples from the sites had low dissolved-solids concentrations (94 to $221 \mathrm{mg} / \mathrm{L}$ ) and low concentrations of trace elements and uranium.

The four sites near Betatakin and Keet Seel Ruins had lower dissolved-solids concentrations (94 to $136 \mathrm{mg} / \mathrm{L}$ ) than the two sites near Inscription House
Ruin (189 to $221 \mathrm{mg} / \mathrm{L}$ ). Although water samples were collected about $50 \mathrm{ft}$ apart for Inscription House Spring and Navajo Creek tributary, the two sites had slightly different water chemistry. Samples from Inscription House Spring had a lower concentration of dissolved oxygen and higher concentrations of sodium, manganese, and ammonia nitrogen than samples from Navajo Creek tributary (table 2).

Concentrations of nitrogen and bacteria were used as indicators of contamination from animal or human wastes (U.S. Geological Survey, 1999; U.S. Environmental Protection Agency, 2002). Nitrogen is naturally present in surface water and ground water, so the concentration has to be elevated above the natural concentration to indicate contamination. Data from recent $\mathrm{N}$-aquifer samples from three sites within 20 miles of the monument areas were available for comparison; one sample from 2001 had a nitrate concentration of $1.2 \mathrm{mg} / \mathrm{L}$ (Thomas, 2002a) and two samples from 2002 had nitrate concentrations of 0.90 and $4.3 \mathrm{mg} / \mathrm{L}$ (Thomas, 2002b). Coliform bacteria are naturally present in the environment and feces, so the presence of total coliforms indicates only possible contamination. Fecal coliform and E. coli bacteria come from human or animal fecal waste only, so the presence of those bacteria indicates contamination from human or animal wastes (U.S. Environmental Protection Agency, 2002). 
Table 2. Field measurements, and concentrations of major ions, nutrients, trace elements, and uranium in water samples from selected sites, Navajo National Monument, Arizona, 2001-02

$\left[{ }^{\circ} \mathrm{C}\right.$, degrees Celsius; $\mu \mathrm{S} / \mathrm{cm}$, microsiemens per centimeter at $25^{\circ} \mathrm{C} ; \mathrm{mg} / \mathrm{L}$, milligrams per liter; $\mu \mathrm{g} / \mathrm{L}$, micrograms per liter; $<$, less than; cols. per $100 \mathrm{~mL}$, colonies per 100 milliliters; e in front of bacteria concentration denotes estimated value; $\mathrm{k}$ after bacteria concentration denotes non-ideal number of colonies on counting plate; dashes indicate no data]

\begin{tabular}{|c|c|c|c|c|c|c|}
\hline Site name & $\begin{array}{l}\text { U.S. Geol } \\
\text { identific }\end{array}$ & $\begin{array}{l}\text { gical Survey } \\
\text { ion number }\end{array}$ & Date of sample & $\begin{array}{c}\text { Specific } \\
\text { conductance, } \\
\text { field }(\mu \text { S/cm) }\end{array}$ & pH, field (units) & $\begin{array}{c}\text { Water } \\
\text { temperature, } \\
\text { field }\left({ }^{\circ} \mathrm{C}\right)\end{array}$ \\
\hline \multirow[t]{3}{*}{ Inscription House Spring } & \multirow{3}{*}{\multicolumn{2}{|c|}{364013110491101}} & 09-13-01 & 378 & 7.6 & 20.7 \\
\hline & & & $03-28-02$ & 361 & 7.5 & 13.5 \\
\hline & & & 08-29-02 & 361 & 7.4 & 16.5 \\
\hline \multirow{3}{*}{$\begin{array}{l}\text { Navajo Creek tributary near } \\
\text { Shonto }\end{array}$} & \multirow{3}{*}{\multicolumn{2}{|c|}{364015110491700}} & $09-13-01$ & 319 & 8.2 & 22.0 \\
\hline & & & $03-28-02$ & 357 & 8.1 & 14.7 \\
\hline & & & 08-29-02 & 353 & 8.1 & 21.7 \\
\hline Betatakin headquarters well & \multicolumn{2}{|c|}{364032110324101} & $09-10-01$ & 189 & 7.9 & 13.8 \\
\hline \multirow[t]{2}{*}{ Betatakin Spring } & \multirow{2}{*}{\multicolumn{2}{|c|}{364100110320301}} & $03-27-02$ & 218 & 8.2 & 9.3 \\
\hline & & & $08-28-02$ & 206 & 8.0 & 12.5 \\
\hline \multirow[t]{3}{*}{ Betatakin Ruin Spring } & \multirow{3}{*}{\multicolumn{2}{|c|}{364102110320201}} & $09-12-01$ & 154 & 7.9 & 16.1 \\
\hline & & & $03-27-02$ & 163 & 8.1 & 11.7 \\
\hline & & & $08-28-02$ & 158 & 7.8 & 14.8 \\
\hline \multirow[t]{3}{*}{ Keet Seel Ruin Spring } & \multirow{3}{*}{\multicolumn{2}{|c|}{364534110293801}} & $09-11-01$ & 162 & 7.7 & 14.4 \\
\hline & & & $03-26-02$ & 168 & 7.8 & 9.9 \\
\hline & & & 08-27-02 & 197 & 7.2 & 14.3 \\
\hline Site name & $\begin{array}{l}\text { Date of } \\
\text { sample }\end{array}$ & $\begin{array}{c}\text { Oxygen, } \\
\text { dissolved, field } \\
\text { (mg/L) }\end{array}$ & $\begin{array}{c}\text { Calcium, } \\
\text { dissolved } \\
\text { (mg/L as Ca) }\end{array}$ & $\begin{array}{l}\text { Magnesium, } \\
\text { dissolved } \\
\text { (mg/L as } \mathrm{Mg})\end{array}$ & $\begin{array}{c}\text { Sodium, } \\
\text { dissolved } \\
\text { (mg/L as } \mathrm{Na})\end{array}$ & $\begin{array}{l}\text { Potassium, } \\
\text { dissolved } \\
\text { (mg/L as K) }\end{array}$ \\
\hline \multirow[t]{3}{*}{ Inscription House Spring } & 09-13-01 & --- & 36.8 & 6.2 & 29.7 & 1.9 \\
\hline & $03-28-02$ & 0.8 & 45.4 & 7.2 & 23.9 & 1.6 \\
\hline & 08-29-02 & 0.1 & 35.4 & 5.7 & 30.0 & 1.5 \\
\hline \multirow{3}{*}{$\begin{array}{l}\text { Navajo Creek tributary near } \\
\text { Shonto }\end{array}$} & $09-13-01$ & 5.6 & 39.6 & 11.5 & 15.9 & 5.8 \\
\hline & $03-28-02$ & 8.2 & 49.9 & 9.4 & 8.4 & 2.9 \\
\hline & 08-29-02 & 7.9 & 49.4 & 10.4 & 8.9 & 3.6 \\
\hline Betatakin headquarters well & 09-10-01 & 7.6 & 34.1 & 3.6 & 4.8 & 1.1 \\
\hline \multirow[t]{2}{*}{ Betatakin Spring } & $03-27-02$ & 8.8 & 30.9 & 3.0 & 3.9 & 1.0 \\
\hline & $08-28-02$ & 9.1 & 32.1 & 3.1 & 4.0 & 1.3 \\
\hline \multirow[t]{3}{*}{ Betatakin Ruin Spring } & $09-12-01$ & 9.2 & 24.0 & 2.1 & 3.5 & 1.0 \\
\hline & $03-27-02$ & 8.5 & 25.6 & 2.2 & 3.6 & 0.8 \\
\hline & $08-28-02$ & 8.0 & 25.5 & 2.2 & 3.4 & 1.0 \\
\hline \multirow[t]{3}{*}{ Keet Seel Ruin Spring } & $09-11-01$ & 6.9 & 24.2 & 2.7 & 3.7 & 0.5 \\
\hline & $03-26-02$ & 7.3 & 27.0 & 2.8 & 3.6 & 0.6 \\
\hline & $08-27-02$ & 5.3 & 26.4 & 2.8 & 3.7 & 0.5 \\
\hline
\end{tabular}


Table 2. Field measurements, and concentrations of major ions, nutrients, trace elements, and uranium in water samples from selected sites, Navajo National Monument, Arizona, 2001--2002-Continued

\begin{tabular}{|c|c|c|c|c|c|c|}
\hline Site name & $\begin{array}{l}\text { Date of } \\
\text { sample }\end{array}$ & $\begin{array}{c}\text { Alkalinity, } \\
\text { dissolved, field } \\
\left.\text { (mg/L as } \mathrm{CaCO}_{3}\right)\end{array}$ & $\begin{array}{l}\text { Bicarbonate, } \\
\text { dissolved, field } \\
\text { (mg/L as } \mathrm{HCO}_{3} \text { ) }\end{array}$ & & $\begin{array}{c}\text { Carbonate, } \\
\text { dissolved, field } \\
\text { (mg/L as } \mathrm{CO}_{3} \text { ) }\end{array}$ & $\begin{array}{c}\text { Sulfate, } \\
\text { dissolved } \\
\left(\mathrm{mg} / \mathrm{L} \text { as } \mathrm{SO}_{4}\right)\end{array}$ \\
\hline \multirow[t]{3}{*}{ Inscription House Spring } & 09-13-01 & 169 & 205 & & $<1$ & 22.5 \\
\hline & $03-28-02$ & 179 & 217 & & $<1$ & 12.0 \\
\hline & 08-29-02 & 162 & 197 & & $<1$ & 11.7 \\
\hline \multirow{3}{*}{$\begin{array}{l}\text { Navajo Creek tributary near } \\
\text { Shonto }\end{array}$} & $09-13-01$ & 156 & 186 & & 2 & 17.3 \\
\hline & $03-28-02$ & 167 & 202 & & 1 & 10.8 \\
\hline & 08-29-02 & 149 & 180 & & $<1$ & 8.1 \\
\hline Betatakin headquarters well & 09-10-01 & 105 & 127 & & $<1$ & 3.3 \\
\hline \multirow[t]{2}{*}{ Betatakin Spring } & $03-27-02$ & 91 & 111 & & $<1$ & 3.5 \\
\hline & $08-28-02$ & 91 & 110 & & $<1$ & 3.3 \\
\hline \multirow[t]{3}{*}{ Betatakin Ruin Spring } & $09-12-01$ & 74 & 90 & & $<1$ & --- \\
\hline & $03-27-02$ & 77 & 93 & & $<1$ & 1.6 \\
\hline & $08-28-02$ & 74 & 89 & & $<1$ & 1.5 \\
\hline \multirow[t]{3}{*}{ Keet Seel Ruin Spring } & $09-11-01$ & 86 & 104 & & $<1$ & 1.5 \\
\hline & $03-26-02$ & 87 & 105 & & $<1$ & 1.3 \\
\hline & $08-27-02$ & 81 & 98 & & $<1$ & 1.5 \\
\hline Site name & $\begin{array}{l}\text { Date of } \\
\text { sample }\end{array}$ & $\begin{array}{c}\text { Chloride, } \\
\text { dissolved } \\
\text { (mg/L as CI) }\end{array}$ & $\begin{array}{c}\text { Fluoride, } \\
\text { dissolved } \\
\text { (mg/L as F) }\end{array}$ & $\begin{array}{l}\text { Silica, } \\
\text { issolved } \\
(\mathrm{mg} / \mathrm{L} \\
\text { as } \mathrm{SiO}_{2} \text { ) }\end{array}$ & $\begin{array}{c}\text { Dissolved } \\
\text { solids, residue } \\
\text { at } 180^{\circ} \mathrm{C}(\mathrm{mg} / \mathrm{L})\end{array}$ & $\begin{array}{c}\text { Aluminum, } \\
\text { dissolved } \\
(\mu \mathrm{g} / \mathrm{L} \text { as } \mathrm{Al})\end{array}$ \\
\hline \multirow[t]{3}{*}{ Inscription House Spring } & $09-13-01$ & 5.3 & 0.3 & 16.6 & 221 & $<1$ \\
\hline & $03-28-02$ & 5.2 & .2 & 15.4 & 219 & --- \\
\hline & 08-29-02 & 4.7 & .3 & 14.2 & 189 & --- \\
\hline \multirow{3}{*}{$\begin{array}{l}\text { Navajo Creek tributary near } \\
\text { Shonto }\end{array}$} & 09-13-01 & 9.3 & .2 & 14.1 & 214 & 2 \\
\hline & $03-28-02$ & 6.3 & .1 & 11.9 & 211 & --- \\
\hline & 08-29-02 & 5.5 & .1 & 14.6 & 207 & --- \\
\hline Betatakin headquarters well & 09-10-01 & 3.7 & $<.2$ & 15.7 & 136 & $<1$ \\
\hline \multirow[t]{2}{*}{ Betatakin Spring } & $03-27-02$ & 3.3 & $<.1$ & 12.7 & 121 & --- \\
\hline & $08-28-02$ & 3.3 & $<.1$ & 12.6 & 113 & --- \\
\hline \multirow[t]{3}{*}{ Betatakin Ruin Spring } & $09-12-01$ & --- & --- & --- & --- & $<1$ \\
\hline & $03-27-02$ & 2.3 & $<.2$ & 11.8 & 94 & --- \\
\hline & $08-28-02$ & 2.2 & $<.1$ & 12.1 & 101 & --- \\
\hline \multirow[t]{3}{*}{ Keet Seel Ruin Spring } & $09-11-01$ & 1.9 & $<.2$ & 13.5 & 100 & $<1$ \\
\hline & $03-26-02$ & 2.0 & $<.1$ & 12.0 & 109 & --- \\
\hline & $08-27-02$ & 2.0 & $<.1$ & 13.0 & 104 & --- \\
\hline
\end{tabular}


Table 2. Field measurements, and concentrations of major ions, nutrients, trace elements, and uranium in water samples from selected sites, Navajo National Monument, Arizona, 2001-2002-Continued

\begin{tabular}{|c|c|c|c|c|c|c|}
\hline Site name & $\begin{array}{l}\text { Date of } \\
\text { sample }\end{array}$ & $\begin{array}{l}\text { Antimony, } \\
\text { dissolved } \\
\text { ( } \mu \mathrm{g} / \mathrm{L} \text { as } \mathrm{Sb})\end{array}$ & $\begin{array}{c}\text { Arsenic, } \\
\text { dissolved } \\
\text { ( } \mu \mathrm{g} / \mathrm{L} \text { as As) }\end{array}$ & $\begin{array}{c}\text { Barium, } \\
\text { dissolved } \\
\text { ( } \mu \mathrm{g} / \mathrm{L} \text { as Ba) }\end{array}$ & $\begin{array}{l}\text { Beryllium, } \\
\text { dissolved } \\
\text { ( } \mu \mathrm{g} / \mathrm{L} \text { as } \mathrm{Be})\end{array}$ & $\begin{array}{c}\text { Boron, } \\
\text { dissolved } \\
(\mu \mathrm{g} / \mathrm{L} \text { as B })\end{array}$ \\
\hline \multirow[t]{3}{*}{ Inscription House Spring } & 09-13-01 & $<0.05$ & 1.1 & 182 & $<.06$ & 60 \\
\hline & $03-28-02$ & --- & 1.1 & --- & --- & 60 \\
\hline & 08-29-02 & --- & 1.3 & --- & --- & 110 \\
\hline \multirow{3}{*}{$\begin{array}{l}\text { Navajo Creek tributary near } \\
\text { Shonto }\end{array}$} & 09-13-01 & .12 & 2.5 & 140 & $<.06$ & 60 \\
\hline & 03-28-02 & --- & 1.2 & --- & --- & 40 \\
\hline & 08-29-02 & --- & 1.9 & --- & --- & 40 \\
\hline Betatakin headquarters well & 09-10-01 & $<.05$ & 0.6 & 194 & $<.06$ & $<10$ \\
\hline \multirow[t]{2}{*}{ Betatakin Spring } & $03-27-02$ & --- & .4 & --- & --- & $<10$ \\
\hline & $08-28-02$ & --- & .5 & --- & --- & $<10$ \\
\hline \multirow[t]{3}{*}{ Betatakin Ruin Spring } & $09-12-01$ & $<.05$ & $<.2$ & 91 & $<.06$ & $<10$ \\
\hline & $03-27-02$ & --- & $<.2$ & --- & --- & $<10$ \\
\hline & $08-28-02$ & --- & .2 & --- & --- & $<10$ \\
\hline \multirow[t]{3}{*}{ Keet Seel Ruin Spring } & 09-11-01 & $<.05$ & .3 & 72 & $<.06$ & $<10$ \\
\hline & $03-26-02$ & --- & .4 & --- & --- & $<10$ \\
\hline & $08-27-02$ & --- & .6 & --- & --- & $<10$ \\
\hline Site name & $\begin{array}{l}\text { Date of } \\
\text { sample }\end{array}$ & $\begin{array}{c}\text { Cadmium, } \\
\text { dissolved } \\
\text { ( } \mu \mathrm{g} / \mathrm{L} \text { as Cd) }\end{array}$ & $\begin{array}{l}\text { Chromium, } \\
\text { dissolved } \\
(\mu \mathrm{g} / \mathrm{L} \text { as } \mathrm{Cr})\end{array}$ & $\begin{array}{c}\text { Cobalt, } \\
\text { dissolved } \\
(\mu \mathrm{g} / \mathrm{L} \text { as Co })\end{array}$ & $\begin{array}{c}\text { Copper, } \\
\text { dissolved } \\
(\mu \mathrm{g} / \mathrm{L} \text { as } \mathrm{Cu})\end{array}$ & $\begin{array}{c}\text { Iron, } \\
\text { dissolved } \\
(\mu \mathrm{g} / \mathrm{L} \text { as Fe) }\end{array}$ \\
\hline \multirow[t]{3}{*}{ Inscription House Spring } & $09-13-01$ & $<0.04$ & $<.8$ & .18 & .3 & $<10$ \\
\hline & 03-28-02 & --- & --- & --- & --- & $<10$ \\
\hline & 08-29-02 & --- & --- & --- & --- & 30 \\
\hline \multirow{3}{*}{$\begin{array}{l}\text { Navajo Creek tributary near } \\
\text { Shonto }\end{array}$} & 09-13-01 & $<.04$ & $<.8$ & .13 & 1.1 & $<10$ \\
\hline & $03-28-02$ & --- & --- & --- & --- & $<10$ \\
\hline & 08-29-02 & --- & --- & --- & --- & $<10$ \\
\hline Betatakin headquarters well & $09-10-01$ & $<.04$ & $<.8$ & .04 & $<.2$ & $<10$ \\
\hline \multirow[t]{2}{*}{ Betatakin Spring } & 03-27-02 & --- & --- & --- & --- & $<10$ \\
\hline & $08-28-02$ & --- & --- & --- & --- & $<10$ \\
\hline \multirow[t]{3}{*}{ Betatakin Ruin Spring } & $09-12-01$ & $<.04$ & $<.8$ & .03 & $<.2$ & $<10$ \\
\hline & 03-27-02 & --- & --- & --- & --- & $<10$ \\
\hline & 08-28-02 & --- & --- & --- & --- & $<10$ \\
\hline \multirow[t]{3}{*}{ Keet Seel Ruin Spring } & 09-11-01 & $<.04$ & $<.8$ & .15 & $<.2$ & $<10$ \\
\hline & 03-26-02 & --- & --- & --- & --- & $<10$ \\
\hline & $08-27-02$ & --- & --- & --- & --- & $<10$ \\
\hline
\end{tabular}


Table 2. Field measurements, and concentrations of major ions, nutrients, trace elements, and uranium in water samples from selected sites, Navajo National Monument, Arizona, 2001-2002-Continued

\begin{tabular}{|c|c|c|c|c|c|c|}
\hline Site name & $\begin{array}{l}\text { Date of } \\
\text { sample }\end{array}$ & $\begin{array}{c}\text { Lead, } \\
\text { dissolved } \\
(\mu \mathrm{g} / \mathrm{L} \text { as } \mathrm{Pb})\end{array}$ & $\begin{array}{c}\text { Manganese, } \\
\text { dissolved } \\
\text { ( } \mu \mathrm{g} / \mathrm{L} \text { as } \mathrm{Mn})\end{array}$ & $\begin{array}{c}\text { Molybdenum, } \\
\text { dissolved } \\
(\mu \mathrm{g} / \mathrm{L} \text { as } \mathrm{Mo})\end{array}$ & $\begin{array}{c}\text { Nickel, } \\
\text { dissolved } \\
(\mu \mathrm{g} / \mathrm{L} \text { as } \mathrm{Ni})\end{array}$ & $\begin{array}{c}\text { Silver, } \\
\text { dissolved } \\
(\mu \mathrm{g} / \mathrm{L} \text { as } \mathrm{Ag})\end{array}$ \\
\hline \multirow[t]{3}{*}{ Inscription House Spring } & 09-13-01 & $<0.08$ & 225 & .8 & $<.06$ & $<1$ \\
\hline & 03-28-02 & --- & --- & --- & --- & --- \\
\hline & 08-29-02 & --- & --- & --- & --- & --- \\
\hline \multirow{3}{*}{$\begin{array}{l}\text { Navajo Creek tributary near } \\
\text { Shonto }\end{array}$} & $09-13-01$ & $<.08$ & 13.8 & 1.1 & $<.06$ & $<1$ \\
\hline & 03-28-02 & --- & --- & --- & --- & --- \\
\hline & 08-29-02 & --- & --- & --- & --- & --- \\
\hline Betatakin headquarters well & 09-10-01 & .23 & $<.1$ & $<.2$ & $<.06$ & $<1$ \\
\hline \multirow[t]{2}{*}{ Betatakin Spring } & $03-27-02$ & --- & --- & --- & --- & --- \\
\hline & $08-28-02$ & --- & --- & --- & --- & --- \\
\hline \multirow[t]{3}{*}{ Betatakin Ruin Spring } & $09-12-01$ & $<.08$ & .4 & $<.2$ & $<.06$ & $<1$ \\
\hline & $03-27-02$ & --- & --- & --- & --- & --- \\
\hline & 08-28-02 & --- & --- & --- & --- & --- \\
\hline \multirow[t]{3}{*}{ Keet Seel Ruin Spring } & 09-11-01 & $<.08$ & .2 & $<.2$ & $<.06$ & $<1$ \\
\hline & 03-26-02 & --- & --- & --- & --- & --- \\
\hline & $08-27-02$ & --- & --- & --- & --- & --- \\
\hline Site name & $\begin{array}{l}\text { Date of } \\
\text { sample }\end{array}$ & $\begin{array}{c}\text { Zinc, } \\
\text { dissolved } \\
\text { ( } \mu \mathrm{g} / \mathrm{L} \text { as } \mathrm{Zn})\end{array}$ & $\begin{array}{c}\text { Uranium, } \\
\text { natural, } \\
\text { dissolved } \\
\text { ( } \mu \mathrm{g} / \mathrm{L} \text { as } \mathrm{U})\end{array}$ & $\begin{array}{l}\text { Coliform, total } \\
\text { (cols. per } \\
100 \mathrm{~mL} \text { ) }\end{array}$ & $\begin{array}{l}\text { Coliform, fecal } \\
\text { (cols. per } \\
100 \mathrm{~mL} \text { ) }\end{array}$ & $\begin{array}{c}\text { E. coli } \\
\text { (cols. per } \\
100 \mathrm{~mL} \text { ) }\end{array}$ \\
\hline \multirow[t]{3}{*}{ Inscription House Spring } & 09-13-01 & 2 & .15 & e500 & e190 & 710 \\
\hline & $03-28-02$ & --- & --- & $\mathrm{e} 26 \mathrm{k}$ & $\mathrm{e} 2 \mathrm{k}$ & $\mathrm{e} 3 \mathrm{k}$ \\
\hline & $08-29-02$ & --- & --- & 340 & $\mathrm{e} 3 \mathrm{k}$ & --- \\
\hline \multirow{3}{*}{$\begin{array}{l}\text { Navajo Creek tributary near } \\
\text { Shonto }\end{array}$} & 09-13-01 & $<1$ & 2.8 & 540 & 10 & $<1$ \\
\hline & $03-28-02$ & --- & --- & 390 & $\mathrm{e} 44 \mathrm{k}$ & $\mathrm{e} 270 \mathrm{k}$ \\
\hline & $08-29-02$ & --- & --- & e6,600 & 4,700 & 3,900 \\
\hline Betatakin headquarters well & 09-10-01 & 49 & .59 & $<1$ & $<1$ & $<1$ \\
\hline \multirow[t]{2}{*}{ Betatakin Spring } & $03-27-02$ & --- & --- & e6k & $<1$ & $<1$ \\
\hline & $08-28-02$ & --- & --- & $\mathrm{e} 5 \mathrm{k}$ & $<1$ & $<1$ \\
\hline \multirow[t]{3}{*}{ Betatakin Ruin Spring } & $09-12-01$ & 1 & .12 & e56 & $<1$ & $<1$ \\
\hline & $03-27-02$ & --- & --- & 36 & $<1$ & $<1$ \\
\hline & $08-28-02$ & --- & --- & 92 & $<1$ & $<1$ \\
\hline \multirow[t]{3}{*}{ Keet Seel Ruin Spring } & $09-11-01$ & $<1$ & .21 & e6k & $<1$ & $<1$ \\
\hline & $03-26-02$ & --- & --- & 32 & $<1$ & $\mathrm{e} 4 \mathrm{k}$ \\
\hline & $08-27-02$ & --- & --- & 340 & 41 & $16 \mathrm{k}$ \\
\hline
\end{tabular}


Table 2. Field measurements, and concentrations of major ions, nutrients, trace elements, and uranium in water samples from selected sites, Navajo National Monument, Arizona, 2001-2002-Continued

\begin{tabular}{|c|c|c|c|c|c|}
\hline Site name & $\begin{array}{l}\text { Date of } \\
\text { sample }\end{array}$ & $\begin{array}{l}\text { Nitrogen, nitrite, } \\
\text { dissolved } \\
\text { (mg/L as N) }\end{array}$ & $\begin{array}{c}\text { Nitrogen, } \\
\text { nitrite + nitrate, } \\
\text { dissolved } \\
\text { (mg/L as N) }\end{array}$ & $\begin{array}{c}\text { Nitrogen, ammonia, } \\
\text { dissolved } \\
\text { (mg/L as } \mathrm{N})\end{array}$ & $\begin{array}{l}\text { Phosphorus, ortho, } \\
\text { dissolved } \\
\text { (mg/L as } \mathrm{P} \text { ) }\end{array}$ \\
\hline \multirow[t]{3}{*}{ Inscription House Spring } & 09-13-01 & 0.040 & .10 & 2.2 & .08 \\
\hline & $03-28-02$ & $<.008$ & $<.05$ & 2.5 & .04 \\
\hline & 08-29-02 & $<.008$ & $<.05$ & 2.3 & .10 \\
\hline \multirow{3}{*}{$\begin{array}{l}\text { Navajo Creek tributary near } \\
\text { Shonto }\end{array}$} & $09-13-01$ & $<.006$ & .07 & .16 & $<.02$ \\
\hline & $03-28-02$ & .009 & .33 & .09 & $<.02$ \\
\hline & 08-29-02 & $<.008$ & $<.05$ & .46 & $<.02$ \\
\hline Betatakin headquarters well & $09-10-01$ & $<.006$ & .83 & $<.04$ & $<.02$ \\
\hline \multirow[t]{2}{*}{ Betatakin Spring } & $03-27-02$ & $<.008$ & .89 & $<.04$ & $<.02$ \\
\hline & $08-28-02$ & $<.008$ & .92 & $<.04$ & $<.02$ \\
\hline \multirow[t]{3}{*}{ Betatakin Ruin Spring } & $09-12-01$ & $<.006$ & .37 & $<.04$ & $<.02$ \\
\hline & $03-27-02$ & $<.008$ & .36 & $<.04$ & $<.02$ \\
\hline & $08-28-02$ & $<.008$ & .38 & $<.04$ & $<.02$ \\
\hline \multirow[t]{3}{*}{ Keet Seel Ruin Spring } & 09-11-01 & $<.006$ & .05 & $<.04$ & $<.02$ \\
\hline & $03-26-02$ & $<.008$ & $<.05$ & $<.04$ & $<.02$ \\
\hline & $08-27-02$ & $<.008$ & .06 & $<.04$ & $<.02$ \\
\hline
\end{tabular}

Concentrations of nitrate (as nitrogen) in water samples from the six sites were generally low $(<0.05$ to $0.92 \mathrm{mg} / \mathrm{L}$ ) and within the range of concentrations at other $\mathrm{N}$-aquifer sites within 20 miles of the study area (table 2). All water samples, except the sample from the Betatakin headquarters well, contained total coliform bacteria. Water samples from Inscription House Spring, Navajo Creek tributary, and Keet Seel Ruin Spring contained indicators of human or animal wastes-fecal coliform and E. coli bacteria. No fecal coliform or $E$. coli bacteria were found in water samples from Betatakin Ruin Spring or Betatakin Spring.

Concentrations of analyzed constituents in water samples from the six sites (tables $\mathbf{2}$ and $\mathbf{3}$ ) were compared to U.S. Environmental Protection Agency Primary and Secondary Maximum Contaminant Levels (U.S. Environmental Protection Agency, 2002; table 3).
No Maximum Contaminant Levels (MCLs) were exceeded. The Secondary Maximum Contaminant Level (SMCL) for manganese $(50 \mu \mathrm{g} / \mathrm{L})$ was exceeded in the September 13, 2001, sample from Inscription House Ruin Spring. No other SMCLs were exceeded.

Water temperatures at all sites varied seasonally; temperatures were cooler in March than in August or September (table 2). There were small seasonal changes in concentrations of major ions, alkalinity, and nutrients. Bacteria concentrations varied substantially (table 2), but that is typical for water that is exposed to air and contamination sources (U.S. Environmental Protection Agency, 2002). Discharge varied seasonally at Navajo Creek tributary, Betatakin Spring, and Betatakin Ruin Spring (table 4). Discharges were too small to accurately measure at Inscription House Spring and Keet Seel Ruin Spring. 
Table 3 U.S. Environmental Protection Agency Primary and Secondary Maximum Contaminant Levels (U.S. Environmental Protection Agency, 2002) for analyzed constituents in water samples from selected sites, Navajo National Monument, Arizona, 2001-02

[Maximum Contaminant Levels (MCLs) are legally enforceable standards that apply to public water systems. MCLs protect public health by limiting the levels of contaminants in drinking water. Secondary Maximum Contaminant Levels (SMCLs) are non-enforceable guidelines regulating contaminants that may cause cosmetic effects (such as skin or tooth discoloration) or aesthetic effects (such as taste, odor, or color) in drinking water. ---, no standard; cols. per $100 \mathrm{~mL}$, colonies per 100 milliliters; mg/L, milligrams per liter; $\mu \mathrm{g} / \mathrm{L}$, micrograms per liter]

\begin{tabular}{|c|c|c|c|c|}
\hline Constituent & Units & MCL & SMCL & Remarks \\
\hline $\mathrm{pH}$ & standard units & --- & $6.5-8.5$ & $\begin{array}{l}\text { SMCL is exceeded when } \mathrm{pH} \text { is below } \\
6.5 \text { or above } 8.5\end{array}$ \\
\hline Total coliform bacteria & $\begin{array}{r}\text { number of } \\
\text { samples }\end{array}$ & 1 & --- & $\begin{array}{l}\text { Not a health threat in itself; used to } \\
\text { indicate whether other potentially } \\
\text { harmful bacteria are present }{ }^{1}\end{array}$ \\
\hline Fecal coliform bacteria & $\begin{array}{r}\text { number of } \\
\text { samples }\end{array}$ & 1 & --- & $\begin{array}{l}\text { From human or animal fecal waste } \\
\quad \text { material }^{1}\end{array}$ \\
\hline Escherichia coli bacteria (E. coli) & $\begin{array}{r}\text { number of } \\
\text { samples }\end{array}$ & 1 & --- & $\begin{array}{l}\text { From human or animal fecal waste } \\
\quad \text { material }^{1}\end{array}$ \\
\hline Sulfate & $\mathrm{mg} / \mathrm{L}$ & --- & 250 & \\
\hline Chloride & $\mathrm{mg} / \mathrm{L}$ & --- & 250 & \\
\hline Fluoride & $\mathrm{mg} / \mathrm{L}$ & 4 & 2 & \\
\hline Dissolved solids & $\mathrm{mg} / \mathrm{L}$ & --- & 500 & \\
\hline Nitrogen, nitrite & $\mathrm{mg} / \mathrm{L}$ & 1 & --- & \\
\hline Nitrogen, nitrite + nitrate & $\mathrm{mg} / \mathrm{L}$ & 10 & --- & \\
\hline Aluminum & $\mu \mathrm{g} / \mathrm{L}$ & --- & 50 to 200 & \\
\hline Antimony & $\mu \mathrm{g} / \mathrm{L}$ & 6 & --- & \\
\hline Arsenic & $\mu \mathrm{g} / \mathrm{L}$ & 10 & --- & \\
\hline Barium & $\mu \mathrm{g} / \mathrm{L}$ & 2,000 & --- & \\
\hline Beryllium & $\mu \mathrm{g} / \mathrm{L}$ & 4 & --- & \\
\hline Cadmium & $\mu \mathrm{g} / \mathrm{L}$ & 5 & --- & \\
\hline Chromium & $\mu \mathrm{g} / \mathrm{L}$ & 100 & --- & \\
\hline Copper & $\mu \mathrm{g} / \mathrm{L}$ & $\begin{array}{r}1,300 \\
\text { action level }\end{array}$ & 1,000 & $\begin{array}{l}\text { Regulated by a treatment technique that } \\
\text { requires systems to control the } \\
\text { corrosiveness of water }\end{array}$ \\
\hline Iron & $\mu \mathrm{g} / \mathrm{L}$ & --- & 300 & \\
\hline Lead & $\mu \mathrm{g} / \mathrm{L}$ & $\begin{array}{r}15 \\
\text { action level }^{2}\end{array}$ & --- & $\begin{array}{l}\text { Regulated by a treatment technique that } \\
\text { requires systems to control the } \\
\text { corrosiveness of water }\end{array}$ \\
\hline Manganese & $\mu \mathrm{g} / \mathrm{L}$ & --- & 50 & \\
\hline Silver & $\mu \mathrm{g} / \mathrm{L}$ & --- & 100 & \\
\hline Zinc & $\mu \mathrm{g} / \mathrm{L}$ & --- & 5,000 & \\
\hline Uranium & $\mu \mathrm{g} / \mathrm{L}$ & 30 & --- & \\
\hline
\end{tabular}

\footnotetext{
${ }_{2}^{1}$ Under the MCL standards for water systems from which fewer than 40 samples are collected per month, no more than one sample may be positive.

2 If more than 10 percent of water samples exceed the action level, water systems must take additional steps.
} 
Table 4. Discharge at selected sites, Navajo National Monument, Arizona, 2001-02

$[<$, less than $]$

\begin{tabular}{lcc}
\hline \multicolumn{1}{c}{ Site name } & Date & $\begin{array}{c}\text { Discharge, } \\
\text { in gallons } \\
\text { per minute }\end{array}$ \\
\hline Inscription House Spring & $09-13-01$ & $<0.5$ \\
& $03-28-02$ & $<0.5$ \\
Navajo Creek tributary near Shonto & $08-29-02$ & $<0.5$ \\
& $09-13-01$ & 22.4 \\
Betatakin Spring & $03-28-02$ & 76.3 \\
Betatakin Ruin Spring & $08-29-02$ & 13.5 \\
& $03-27-02$ & 0.90 \\
Keet Seel Ruin Spring & $08-28-02$ & 2.7 \\
& $09-12-01$ & 0.76 \\
& $03-27-02$ & 0.90 \\
& $08-28-02$ & 0.90 \\
& $09-11-01$ & $<0.5$ \\
& $03-26-02$ & $<0.5$ \\
& $08-27-02$ & $<0.5$ \\
\hline
\end{tabular}

\section{SUMMARY}

The USGS, in cooperation with the National Park Service, collected and analyzed water samples from six sites at Navajo National Monument in northeastern Arizona during September 2001 to August 2002. This inventory was part of a nationwide program of the National Park Service to obtain baseline water-quality information in national parks and monuments throughout the United States. The water-quality data and applicable U.S. Environmental Protection Agency MCLs and SMCLs are presented in this report.

Navajo National Monument is within the Navajo Indian Reservation and encloses three ancient Indian ruins in three separate areas. The total area of the monument is 0.56 square mile. Water samples were collected from two springs and one well near Betatakin Ruin, one spring near Keet Seel Ruin, and one spring and one stream near Inscription House Ruin. Water from all the sites is from the $\mathrm{N}$ aquifer-a regional sandstone aquifer that is the source of drinking water for most members of the Navajo Nation and Hopi Tribe in northeastern Arizona.
Water-quality samples were collected in September 2001, March 2002, and August 2002 from the two sites near Inscription House Ruin, from one spring near Betatakin Ruin, and from one spring near Keet Seel Ruin. Samples were collected in March 2002 and August 2002 from one spring near Betatakin Ruin. One sample was collected in September 2001 from the well near Betatakin Ruin. The samples were analyzed for different sets of constituents depending on the site and the frequency of sampling.

Concentrations of dissolved solids, major ions, trace elements, and uranium were low at the six sites. Dissolved-solids concentrations ranged from 94 to 221 $\mathrm{mg} / \mathrm{L}$. Concentrations of nitrate (as nitrogen) were generally low $(<0.05$ to $0.92 \mathrm{mg} / \mathrm{L})$ and within the range of concentrations at other $\mathrm{N}$-aquifer sites within 20 miles of the study area. Water samples from Inscription House Spring, Navajo Creek tributary (near Inscription House Ruin), and Keet Seel Ruin Spring contained indicators of human or animal wastes-fecal coliform and E. coli bacteria.

\section{REFERENCES CITED}

Britton, L.J., and Greeson, P.E., 1988, Methods for collection and analysis of aquatic biological and microbiological samples: U.S. Geological Survey Techniques of Water-Resources Investigations, book 5, chap. A4, 685 p.

Davis, G.E., Hardt, W.F., Thompson, L.K., and Cooley, M.E., 1963, Records of ground-water supplies, part 1, of geohydrologic data in the Navajo and Hopi Indian Reservations, Arizona, New Mexico, and Utah: Arizona State Land Department Water-Resources Report 12-A, $159 \mathrm{p}$.

Faires, L.M., 1993, Methods of analysis by the U.S. Geological Survey National Water Quality Laboratory-Determination of metals in water by inductively coupled plasma-mass spectrometry: U.S. Geological Survey Open-File Report 92-634, 28 p.

Fishman, M.J, 1993, Methods of analysis by the U.S. Geological Survey National Water Quality Laboratory - Determination of inorganic and organic constituents in water and fluvial sediments: U.S. Geological Survey Open-File Report 93-125, 217 p. 
Fishman, M.J., and Friedman, L.C., eds., 1989, Methods for the determination of inorganic substances in water and fluvial sediments: U.S. Geological Survey Techniques of Water-Resources Investigations, book 5, chap. A1, $545 \mathrm{p}$.

Friedman, L.C., and Erdmann, D.E., 1982, Quality assurance practices for the chemical and biological analyses of water and fluvial sediments: U.S. Geological Survey Techniques of Water-Resources Investigations, book 5, chap. A6, 181 p.

Garbarino, J.R., 1999, Methods of analysis by the U.S. Geological Survey National Water Quality Laboratory-Determination of dissolved arsenic, boron, lithium, selenium, strontium, thallium, and vanadium using inductively coupled plasma-mass spectrometry: U.S. Geological Survey Open-File Report 99-093, 31 p.

Jones, S.R., and Garbarino, J.R., 1999, Methods of analysis by the U.S. Geological Survey National Water Quality Laboratory-Determination of arsenic and selenium in water and sediment using graphite furnace atomic absorption spectrometry: U.S. Geological Survey Open-File Report 98-639, 39 p.

Koterba, M.T., Wilde, F.D., and Lapham, W.W., 1995, Ground-water data-collection protocols and procedures for the National Water-Quality Assessment ProgramCollection and documentation of water-quality samples and related data: U.S. Geological Survey Open-File Report 95-399, 113 p.

McLain, B.J., 1993, Methods of analysis by the U.S. Geological Survey National Water Quality Laboratory-Determination of chromium in water by graphite furnace atomic absorption spectrophotometry: U.S. Geological Survey Open-File Report 93-449, $16 \mathrm{p}$.

Myers, D.N., and Wilde, F.D., eds., 1999, National field manual for the collection of water-quality dataBiological indicators: U.S. Geological Survey Techniques of Water-Resources Investigations, book 9, chap. A7, v.p.

Struzeski, T.M., DeGiacoma, W.J., and Zayhowski, E.J., 1996, Methods of analysis by the U.S. Geological Survey National Water Quality LaboratoryDetermination of dissolved aluminum and boron in water by inductively coupled plasma-atomic emission spectrometry: U.S. Geological Survey Open-File Report 96-149, 17 p.
Thomas, B.E., 2002a, Ground-water, surface-water, and water-chemistry data, Black Mesa area, northeastern Arizona-2000-2001, and performance and sensitivity of the 1988 USGS numerical model of the N aquifer: U.S. Geological Survey Water-Resources Investigations Report 02-4211, 75 p.

-2002b, Ground-water, surface-water, and waterchemistry data, Black Mesa area, northeastern Arizona-2001-02: U.S. Geological Survey Open-File Report 02-485, 43 p.

U.S. Department of Agriculture, Natural Resources Conservation Service, 1999, Arizona annual precipitation: Fort Worth, Texas, USDA-NRCS National Cartography and Geospatial Center, scale 1:1,300,000.

U.S. Environmental Protection Agency, 2002, Current drinking water standards, National Primary and Secondary Drinking Water Regulations: Washington, D.C., U.S. Environmental Protection Agency, accessed October 16, 2002, at URL http://www.epa.gov/safewater/mcl.html.

U.S. Geological Survey, 1999, The quality of our nation's waters-Nutrients and pesticides: U.S. Geological Survey Circular 1225, $82 \mathrm{p}$.

Western Regional Climate Center, Desert Research Institute, 2002, Arizona climate summaries: Reno, Nevada, Western Regional Climate Center, Desert Research Institute, accessed October 3, 2002, at URL http://www.wrcc.dri.edu/summary/climsmaz.html.

Wilde, F.D., and Radtke, D.B., eds., 1998, National field manual for the collection of water-quality data-Field measurements: U.S. Geological Survey Techniques of Water-Resources Investigations, book 9, chap. A6, v.p.

Wilde, F.D., Radtke, D.B., Gibs, Jacob, and Iwatsubo, R.T., 1998a, National field manual for the collection of water-quality data-Preparations for water sampling: U.S. Geological Survey Techniques of Water-Resources Investigations, book 9, chap. A1, 38 p.

1998b, National field manual for the collection of water-quality data-Selection of equipment for water sampling: U.S. Geological Survey Techniques of Water-Resources Investigations, book 9, chap. A2, 82 p.

$1998 \mathrm{c}$, National field manual for the collection of water-quality data-Cleaning of equipment for water sampling: U.S. Geological Survey Techniques of Water-Resources Investigations, book 9, chap. A3, 65 p.

1999, National field manual for the collection of water-quality data-Collection of water samples: U.S. Geological Survey Techniques of Water-Resources Investigations, book 9, chap. A4, 103 p. 